\title{
Effects of Inelastic Neutrino-Nucleus Scattering on Supernova Dynamics and Radiated Neutrino Spectra
}

\author{
K. Langanke, ${ }^{1,2}$ G. Martínez-Pinedo, ${ }^{1}$ B. Müller, ${ }^{3}$ H.-Th. Janka, ${ }^{3}$ \\ A. Marek, ${ }^{3}$ W.R. Hix, ${ }^{4}$ A. Juodagalvis, ${ }^{5}$ and J.M. Sampaio ${ }^{6}$ \\ ${ }^{1}$ Gesellschaft für Schwerionenforschung Darmstadt, Planckstr. 1, D-64259 Darmstadt, Germany \\ ${ }^{2}$ Institut für Kernphysik, TU Darmstadt, Schlossgartenstr. 9, D-64289 Darmstadt, Germany \\ ${ }^{3}$ Max-Planck-Institut für Astrophysik, Karl-Schwarzschild-Str. 1, D-85741 Garching, Germany \\ ${ }^{4}$ Physics Division, Oak Ridge National Laboratory, Oak Ridge, TN 37831, USA \\ ${ }^{5}$ Institute of Theoretical Physics and Astronomy, A. Gostauto St. 12, 01108 Vilnius, Lithuania \\ ${ }^{6}$ Centro de Física Nuclear da Univ. de Lisboa, Av. Prof. Gama Pinto 2, P-1649-003 Lisboa, Portugal
}

(Dated: October 24, 2018)

\begin{abstract}
Based on the shell model for Gamow-Teller and the Random Phase Approximation for forbidden transitions, we have calculated reaction rates for inelastic neutrino-nucleus scattering (INNS) under supernova (SN) conditions, assuming a matter composition given by Nuclear Statistical Equilibrium. The rates have been incorporated into state-of-the-art stellar core-collapse simulations with detailed energy-dependent neutrino transport. While no significant effect on the SN dynamics is observed, INNS increases the neutrino opacities noticeably and strongly reduces the high-energy tail of the neutrino spectrum emitted in the neutrino burst at shock breakout. Relatedly the expected event rates for the observation of such neutrinos by earthbound detectors are reduced by up to about $60 \%$.
\end{abstract}

PACS numbers: 97.60.Bw, 26.50.+x, 25.30.Pt, 95.55.Vj

The simulation of core-collapse supernovae $(\mathrm{SNe})$ is one of the great astrophysical challenges requiring sophisticated computational skills and detailed input from various fields of physics (e.g., [1, 2]). It is well known that processes mediated by the weak interaction play an essential role for the collapse dynamics and the explosion mechanism 3, 4, 5]. However, inelastic neutral-current neutrino scattering on nuclei (INNS), $A+\nu \rightarrow A^{\star}+\nu^{\prime}$ where $A, A^{\star}$ describe the nucleus with mass number $A$ before and after the scattering process, has not yet been satisfactorily considered in SN simulations. In this process energy is exchanged between neutrinos and matter, and hence it can potentially contribute to SN physics by (i) speeding up the thermalization of neutrinos with matter after neutrino trapping during the collapse phase, (ii) changing the neutrino opacity which will in turn modify the spectra of neutrinos released in the SN and to be observed by earthbound detectors, (iii) preheating the matter outside the shock front after bounce before arrival of the shock [] ], (iv) reviving the stalled shock, and (v) spallating nucleons from nuclei and thus modifying explosive nucleosynthesis. In this Letter we will report about SN simulations which consider INNS reactions and allow us to explore topics (i)-(iii) in detail.

The effect of INNS in SN simulations has been investigated previously in an exploratory study 7], approximating the matter composition by a representative nucleus, ${ }^{56} \mathrm{Fe}$. The reaction cross sections were based on a nuclear model for temperature $T=0$, combining a truncated shell model evaluation of the allowed Gamow-Teller (GT) response to the cross section with estimates of forbidden components derived from the Goldhaber-Teller model. The study concluded that INNS rates can com- pete with those of neutrino-electron scattering at moderate and high neutrino energies $(E>25 \mathrm{MeV})$, while they are significantly smaller for low $E$. No significant effects of INNS on the stalled shock by preheating the accreted matter were found in [7].

Approximating the composition of SN matter by the ground state of the even-even nucleus ${ }^{56} \mathrm{Fe}$ is too simple an assumption for the calculation of INNS interaction rates. At low and moderate neutrino energies, this neutral-current process is dominated by GT transitions mediated by the operator $\mathrm{GT}_{0}=\boldsymbol{\sigma} \boldsymbol{\tau}_{0}$, where $\boldsymbol{\tau}_{ \pm}, \boldsymbol{\tau}_{0}$ are the components of the isospin operator in spherical coordinates. The spin operator $\boldsymbol{\sigma}$ can change the angular momentum of the initial state $J_{i}$ by one unit and hence connects the ${ }^{56} \mathrm{Fe}$ ground state with spin/parity $J_{i}=0^{+}$ only to final states in the same nucleus with $J_{f}=1^{+}$. As the lowest $1^{+}$state in ${ }^{56} \mathrm{Fe}$ is at an excitation energy of $E_{x}=3 \mathrm{MeV}$, there exists a threshold for inelastic neutrino scattering on ${ }^{56} \mathrm{Fe}$ (similarly on other even-even nuclei) and the cross sections are rather small for low neutrino energies.

Supernova matter consists of a mixture of many nuclei with even and odd proton and neutron numbers. Since odd- $A$ and odd-odd (i.e., with odd proton and neutron numbers) nuclei miss the strong pairing gap that lowers the ground state in even-even nuclei relative to excited states, and have usually $J_{i} \neq 0$, GT transitions from the ground state to levels at rather low excitation energies are possible, reducing the threshold for inelastic neutrino scattering on the ground state and generally increasing the cross sections at low E. More importantly, $\mathrm{SN}$ matter has a non-zero temperature of order $1 \mathrm{MeV}$ or higher, requiring the description of nuclei as a thermal 
ensemble. This completely removes the energy threshold for inelastic neutrino scattering, because nuclei are with non-vanishing probability in excited states that can be connected to the ground state or final states at smaller excitation energies. Such scattering events correspond to de-excitation of the nucleus with the consequence that the final neutrino energy $E^{\prime}$ is larger than the initial energy $E$. In fact, it has been demonstrated in [8] that the consideration of $T \neq 0$ effects can drastically increase the INNS cross sections for low neutrino energies.

Our strategy to calculate the INNS cross sections appropriate for SN conditions is based on the fact that the matter composition at the relatively high temperatures $(T \gtrsim 1 \mathrm{MeV})$ is given by Nuclear Statistical Equilibrium (NSE). The computation of cross sections for individual nuclei was described in [9], where the allowed GT transitions for inelastic neutrino scattering on the ground states were calculated within the shell model. Due to the absence of experimental data for INNS this approach was validated by detailed comparison to precision M1 data from inelastic electron scattering, which for spherical nuclei is dominated by transitions mediated by an operator proportional to $\mathrm{GT}_{0}$ [10]. The contributions from forbidden transitions, which become increasingly important at moderate and high $E$, were evaluated within the Random Phase Approximation (RPA). To incorporate the $T \neq 0$ effects, our treatment distinguishes between 'upscattering' (i.e. the final neutrino energy is smaller than the initial one; $\left.E^{\prime}<E\right)$ and 'down-scattering' $\left(E^{\prime}>E\right)$ contributions to the cross sections. Up-scattering was treated approximately assuming the Brink hypothesis, i.e. assuming that the $\mathrm{GT}_{0}$ and forbidden distributions on the excited states are the same as calculated for the ground state. The down-scattering contribution is obtained from the up-scattering contribution using detailed balance.

In a SN environment the relevant INNS cross section $d \sigma\left(E, E^{\prime}\right) / d E^{\prime}$ is obtained by folding the cross sections $d \sigma_{i}\left(E, E^{\prime}\right) / d E^{\prime}$ for individual nuclei with the appropriate abundance distributions, $d \sigma\left(E, E^{\prime}\right) / d E^{\prime}=$ $\sum_{i} Y_{i} d \sigma_{i}\left(E, E^{\prime}\right) / d E^{\prime}=\left(\sum_{i} Y_{i}\right)\left\langle d \sigma\left(E, E^{\prime}\right) / d E^{\prime}\right\rangle$, where the sum runs over all nuclei present and $Y_{i}$ denotes the abundance $n_{i} / n_{b}$ of a given species $\left(n_{i}\right.$ and $n_{b}$ being the number densities of nuclei and baryons, respectively). Since the temperature is sufficiently high $(T \gtrsim 1 \mathrm{MeV})$ once the inelastic process becomes relevant, the nuclear composition is well approximated by NSE and, like in [4], we have calculated the abundances $Y_{i}$ from a Saha-like NSE distribution, including Coulomb corrections [11, 12].

Reference [9] has presented detailed cross sections for inelasic neutrino scattering on about 50 nuclei of the $Z=25-28$ isotope chains. Additionally, using the method described above we have also included cross sections for the $\mathrm{Cr}(Z=24)$ isotopic chain. Importantly, for $T \gtrsim 1 \mathrm{MeV}$, all these calculations show little variations between individual cross sections. Therefore, we have assumed that the average cross section over the full composition can be approximated by $\left\langle d \sigma\left(E, E^{\prime}\right) / d E^{\prime}\right\rangle \approx$ $\sum_{i}^{\prime} Y_{i} d \sigma_{i}\left(E, E^{\prime}\right) / d E^{\prime} / \sum_{i}^{\prime} Y_{i}$, where the sum is restricted to the pool of nuclei for which individual cross sections have been calculated in [9]. We have determined a cross section table for a large variety of temperatures $(0.517 \leq T[\mathrm{MeV}] \leq 3.447)$, densities $\left(10^{8} \leq \rho\left[\mathrm{g} \mathrm{cm}^{-3}\right] \leq\right.$ $\left.6.31 \times 10^{12}\right)$, and electron-to-baryon ratios $\left(Y_{e}\right.$, chosen density-dependent between 0.23 and 0.55 ), for a mesh of initial and final neutrino energies between zero and $100 \mathrm{MeV}$.

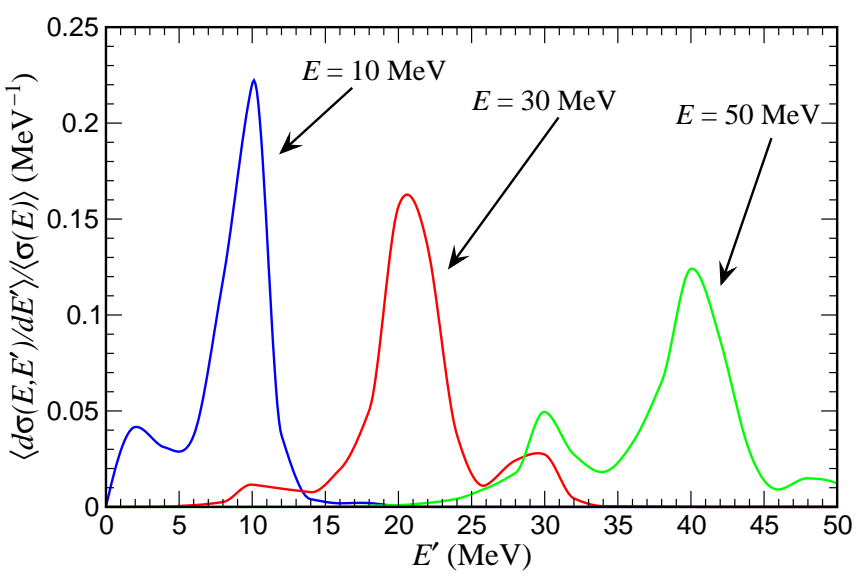

FIG. 1: Normalized final-energy neutrino spectra for initial neutrino energies of 10,30 , and $50 \mathrm{MeV}$ and $T=1 \mathrm{MeV}$, $\rho=6 \times 10^{11} \mathrm{~g} \mathrm{~cm}^{-3}$ and $Y_{e}=0.397$. The total composition averaged cross section, $\langle\sigma(E)\rangle$, for these energies is $0.48 \times 10^{-42}, 38 \times 10^{-42}, 243 \times 10^{-42} \mathrm{~cm}^{2}$, respectively. For conditions of $T=2 \mathrm{MeV}, \rho=2.5 \times 10^{12} \mathrm{~g} \mathrm{~cm}^{-3}$ and $Y_{e}=0.275$, the total composition averaged cross section is $0.97 \times 10^{-42}, 41 \times 10^{-42}, 267 \times 10^{-42} \mathrm{~cm}^{2}$ for the same energies, showing a slight dependence on conditions.

Figure 1 shows the normalized final-energy neutrino spectra at $T=1 \mathrm{MeV}, \rho=6 \times 10^{11} \mathrm{~g} \mathrm{~cm}^{-3}$ and $Y_{e}=$ 0.397 for three different initial energies. For $E \lesssim 10 \mathrm{MeV}$ down-scattering contributes significantly to the cross section; i.e., the de-excitation of thermally populated nuclear levels produces neutrinos with $E^{\prime}>10 \mathrm{MeV}$. Downscattering becomes essentially irrelevant at higher neutrino energies. For $E=30 \mathrm{MeV}$ the cross section is dominated by the excitation of the $\mathrm{GT}_{0}$ centroid, giving rise to a cross section peak around $E^{\prime}=20 \mathrm{MeV}$. For even higher neutrino energies forbidden transitions contribute noticeably to the cross section. The peaks for $E=50 \mathrm{MeV}$ correspond to excitations of the centroids of the $\mathrm{GT}_{0}$ and dipole transition strengths.

The SN calculations presented in this work were performed in spherical symmetry with the neutrinohydrodynamics code VERTEx (for details, see [13, 14]). The code module that integrates the nonrelativistic hydrodynamics equations is a conservative, Eulerian implementation of a Godunov-type scheme with higher-order spatial and temporal accuracy. The self-gravity of the 
stellar gas is treated with an approximation to general relativity as discussed in [15] and tested against fully relativistic simulations in [15, 16]. The time-implicit transport routine solves the moment equations for neutrino number, energy, and momentum. It employs a variable Eddington closure factor that is obtained from iterating to convergence a simplified Boltzmann equation coupled to the set of its moment equations. A state-ofthe-art description of the interactions of neutrinos $(\nu)$ and antineutrinos $(\bar{\nu})$ of all flavors is included according to $14,17,18]$.

We compare here the effects of INNS by simulating the collapse of a $15 M_{\odot}$ progenitor star (model s15a28 of [19]) with three different nuclear equations of state (EoSs) from Lattimer \& Swesty (LS) [20], Shen et al. [21], and Hillebrandt \& Wolff 22], which are applied above some minimum density $\left(\sim 10^{8} \mathrm{~g} \mathrm{~cm}^{-3}\right.$ before shock breakout and $10^{11} \mathrm{~g} \mathrm{~cm}^{-3}$ afterwards). At lower densities a mixture of ideal gases of $\mathrm{e}^{ \pm}$, photons, nucleons, and nuclei is used, and changes of the chemical composition are determined by nuclear burning or through a 17 -species NSE network. The high-density EoSs yield different timevariable abundances of neutrons, protons, $\alpha$-particles, and a representative heavy nucleus, whose charge and mass numbers $(Z, A)$ also differ between the three considered cases. As a consequence, the INNS rates for $\nu$ and $\bar{\nu}$ of all flavors are computed as the product of the tabulated pool-averaged differential cross sections, $\left\langle d \sigma\left(E, E^{\prime}\right) / d E^{\prime}\right\rangle$, and an EoS-dependent abundance $Y_{A}$ of the representative heavy nucleus. Tests showed that insignificant changes of the SN results for a given EoS occur when the factor $Y_{A}$ in the rate calculation is replaced by the abundance sum, $\sum_{i} Y_{i}$, of all species of the NSE distribution. For reducing the dimensionality of the table, information of the cross section variation with the scattering angle was not stored. In the hydrodynamic simulations we therefore made the approximation that neutrinos colliding inelastically with nuclei are redistributed isotropically.

The effects of INNS on the SN evolution and the properties of the emitted neutrinos turn out to be very similar for all three employed nuclear EoSs. Despite causing a higher neutrino opacity by additional neutrino-nuclei interactions, INNS leads to a slightly stronger deleptonization and to a very small increase of the entropy in the homologously collapsing inner core. These effects are caused by up-scattering reactions in which high-energy neutrinos from electron captures lose part of their energy, thus producing additional heating of the stellar matter and escaping faster from the stellar interior because neutrinos with lower energies possess a much smaller total interaction probability. The additional deleptonization, however, is so tiny that it reduces the collapse time to bounce and the enclosed mass of the shock formation radius only on a miniscule level. In contrast, due to the additional opacity, the number of electron neutri- nos radiated in the luminous burst that is released when the shock breaks out from the neutrino-opaque to the neutrino-transparent regime, is slightly smaller (on the third digit) when INNS is included. Moreover, these reactions increase the neutrino-matter coupling and thus the total energy transfer rate in non-conservative scatterings (mainly on electrons and nuclei) ahead of the shock by typically a factor of $2-3$ during the first $\lesssim 50 \mathrm{~ms}$ after core bounce (before the shock reaches a radius where the preshock density drops below the minimum value of our cross section table so that INNS was not taken into account any longer). Nevertheless, the preshock heating rates are large for too short a time to lead to consequences for the shock propagation and SN dynamics. The differences with and without INNS remain smaller than the numerical resolution limit during all the simulated postbounce evolution.

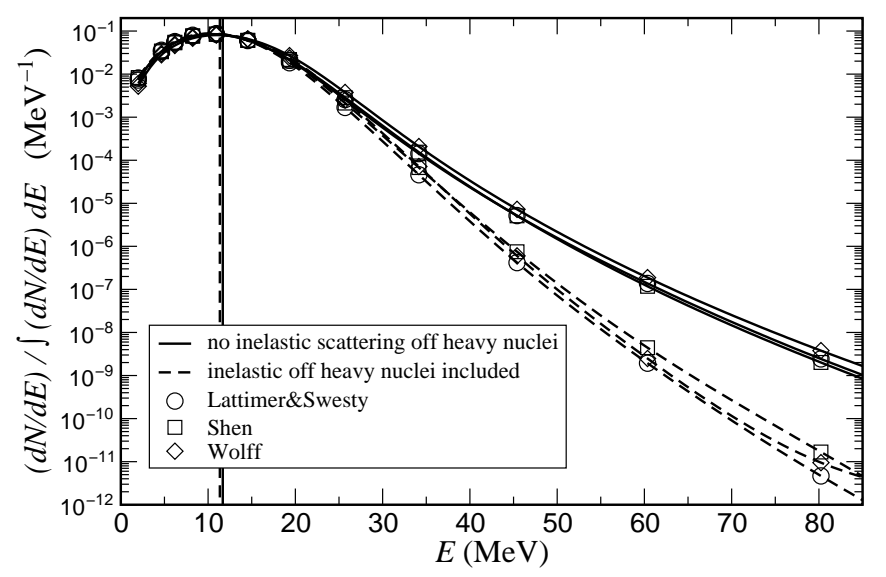

FIG. 2: Normalized $\nu_{\mathrm{e}}$ number spectra radiated during the shock breakout burst as seen by a distant observer at rest. Results are shown for simulations with the three different nuclear EoSs employed in this work. For better comparison of the strongly time-dependent spectra during this evolution phase, integration in a window of $8 \mathrm{~ms}$ around the peak luminosity was performed. Inelastic neutrino scattering off nuclei (dashed lines) leads mostly to energy losses of high-energy neutrinos and thus reduces the high-energy tails of the spectra. The vertical lines mark the mean spectral energies.

The most significant impact of INNS on the radiated neutrino spectra occurs in a time interval of about $15 \mathrm{~ms}$ around the shock breakout $\nu_{\mathrm{e}}$ burst, when the preshock matter, which is composed mainly of heavy nuclei in NSE, has still a high density and therefore a fairly large optical depth for the escaping neutrinos. For this reason, high-energy neutrinos are efficiently degraded in energy space by frequent inelastic collisions with nuclei. While the mean spectral energy is reduced only by a modest amount $(0.4-0.5 \mathrm{MeV})$, the high-energy tail of the emitted $\nu_{\mathrm{e}}$ burst spectrum is strongly suppressed. The normalized spectra of electron neutrinos during a time interval of $8 \mathrm{~ms}$ around the maximum luminosity of the burst for simulations with and without INNS are displayed in 
TABLE I: Electron neutrino cross sections for scattering off electrons and for charged-current interactions with nuclei of different detector materials, averaged over the SN spectrum of Fig. 2, using the results obtained with the LS-EoS. The different cross sections were taken from: e [23], d [24], ${ }^{12} \mathrm{C}[25]$, ${ }^{16} \mathrm{O}$ [26], ${ }^{40} \mathrm{Ar}\left[27\right.$ ], ${ }^{56} \mathrm{Fe}$ and ${ }^{208} \mathrm{~Pb}$ [28].

\begin{tabular}{lccc}
\hline \hline Material & \multicolumn{2}{c}{$\langle\sigma\rangle\left(10^{-42} \mathrm{~cm}^{2}\right)$} & Reduction \\
& With INNS & Without INNS & \\
\hline $\mathrm{e}$ & 0.106 & 0.110 & $3 \%$ \\
$\mathrm{~d}$ & 4.92 & 5.36 & $8 \%$ \\
${ }^{12} \mathrm{C}$ & 0.050 & 0.080 & $37 \%$ \\
${ }^{12} \mathrm{C}\left(\mathrm{N}_{\mathrm{gs}}\right)$ & 0.046 & 0.071 & $35 \%$ \\
${ }^{16} \mathrm{O}$ & 0.0053 & 0.0128 & $58 \%$ \\
${ }^{40} \mathrm{Ar}$ & 13.4 & 15.1 & $11 \%$ \\
${ }^{56} \mathrm{Fe}$ & 6.2 & 7.5 & $17 \%$ \\
${ }^{208} \mathrm{~Pb}$ & 103.3 & 124.5 & $17 \%$ \\
\hline \hline
\end{tabular}

Fig. 2. A similar effect of INNS is observed for $\bar{\nu}_{\mathrm{e}}$ and heavy-lepton neutrinos $\nu_{x}$ (see [1]) when their luminosities begin to rise at the time the $\nu_{\mathrm{e}}$ emission comes down from its peak but still remains clearly dominant for some ten milliseconds.

Considering that the neutrino absorption cross sections on nuclei typically increase steeply with $E$, the reduction of the high-energy spectral tail has important consequences for the detectability of the $\nu_{\mathrm{e}}$ burst from $\mathrm{SNe}$ by experiments [29]. This is demonstrated in Table [1 where we compare the relevant $\nu_{\mathrm{e}}$ detection cross sections calculated for the SN spectrum with and without INNS. In both cases the LS EoS was used; the other EoSs yield very similar results. The reduction depends, of course, strongly on the nuclear threshold energy. For ${ }^{12} \mathrm{C}$ and ${ }^{16} \mathrm{O}$, which are detector material in Borexino, MiniBooNe, SNO, and Super-Kamiokande, only neutrinos with relatively high energies $\left(E>17 \mathrm{MeV}\right.$ for ${ }^{12} \mathrm{C}$ and $15 \mathrm{MeV}$ for ${ }^{16} \mathrm{O}$ ) can trigger charged-current reactions; consequently, the change in the SN spectrum reduces the detection cross section by roughly $35 \%$ and nearly $60 \%$, respectively. The data for ${ }^{12} \mathrm{C}\left(\mathrm{N}_{\mathrm{gs}}\right)$ include only the transition to the ${ }^{12} \mathrm{~N}$ ground state. This is the only bound state in ${ }^{12} \mathrm{~N}$ and the easiest transition to detect, because ${ }^{12} \mathrm{~N}$ decays by $\mathrm{e}^{+}$emission after $11 \mathrm{~ms}$. The signal will be a $\mathrm{e}^{+} \mathrm{e}^{-}$annihilation. We find a reduction of $8 \%$ for the $\left(\nu_{\mathrm{e}}, \mathrm{e}^{-}\right)$cross section on deuterons (the main SN detector material in SNO), $11 \%$ for ${ }^{40} \mathrm{Ar}$ (ICARUS), $17 \%$ for ${ }^{56} \mathrm{Fe}$ (Minos) and ${ }^{208} \mathrm{~Pb}$ (OMNIS). In contrast, since the cross section for scattering off electrons increases linearly with $E$, its reduction is relatively small (only 3\%), which is relevant for SNO and SuperKamiokande.

In summary, we have reported about the first SN simulations that include INNS, which allows for an additional mode of energy exchange between neutrinos and mat- ter. We found that this mode has little effect on the collapse dynamics and the shock propagation. However, INNS modifies the radiated neutrino spectra. In particular, INNS strongly reduces the high-energy spectral tail of the $\nu_{\mathrm{e}}$ burst at shock breakout. In turn, this noticeably decreases the cross section for the observation of the burst neutrinos from future $\mathrm{SNe}$ by neutrino detectors.

In Garching, this work was supported by DFG grants SFB/TR 27 and SFB 375. JMS acknowledges a grant from Fundação para a Ciência e Tecnologia.

[1] H.-T. Janka, K. Langanke, A. Marek, G. MartínezPinedo, and B. Müller, Phys. Repts. 442, 38 (2007).

[2] A. Mezzacappa, Ann. Rev. Nucl. Part. Sci. 55, 467 (2005).

[3] H. A. Bethe, Rev. Mod. Phys. 62, 801 (1990).

[4] K. Langanke and G. Martínez-Pinedo, Rev. Mod. Phys. 75, 819 (2003).

[5] A. Burrows, S. Reddy, and T. A. Thompson, Nuclear Physics A 777, 356 (2006), arXiv:astro-ph/0404432.

[6] W. C. Haxton, Phys. Rev. Lett. 60, 1999 (1988).

[7] S. W. Bruenn and W. C. Haxton, Astrophys. J. 376, 678 (1991).

[8] J. M. Sampaio, K. Langanke, G. Martínez-Pinedo, and D. J. Dean, Phys. Lett. B 529, 19 (2002).

[9] A. Juodagalvis, K. Langanke, G. Martínez-Pinedo, W. R. Hix, D. J. Dean, and J. M. Sampaio, Nucl. Phys. A 747, 87 (2005).

[10] K. Langanke, G. Martínez-Pinedo, P. von NeumannCosel, and A. Richter, Phys. Rev. Lett. 93, 202501 (2004).

[11] W. R. Hix, Ph.D. thesis, Harvard University (1995).

[12] E. Bravo and D. García-Senz, Mon. Not. Roy. Ast. Soc. 307, 984 (1999).

[13] M. Rampp and H.-T. Janka, Astron. \& Astrophys. 396, 361 (2002).

[14] R. Buras, M. Rampp, H.-T. Janka, and K. Kifonidis, Astron. \& Astrophys. 447, 1049 (2006).

[15] A. Marek, H. Dimmelmeier, H.-T. Janka, E. Müller, and R. Buras, Astron. \& Astrophys. 445, 273 (2006).

[16] M. Liebendörfer, M. Rampp, H.-T. Janka, and A. Mezzacappa, Astrophys. J. 620, 840 (2005).

[17] K. Langanke, G. Martínez-Pinedo, J. M. Sampaio, D. J. Dean, W. R. Hix, O. E. B. Messer, A. Mezzacappa, M. Liebendörfer, H.-T. Janka, and M. Rampp, Phys. Rev. Lett. 90, 241102 (2003).

[18] A. Marek, H.-T. Janka, R. Buras, M. Liebendörfer, and M. Rampp, Astron. \& Astrophys. 443, 201 (2005).

[19] A. Heger, S. E. Woosley, G. Martínez-Pinedo, and K. Langanke, Astrophys. J. 560, 307 (2001).

[20] J. M. Lattimer and F. D. Swesty, Nucl. Phys. A 535, 331 (1991).

[21] H. Shen, H. Toki, K. Oyamatsu, and K. Sumiyoshi, Nucl. Phys. A 637, 435 (1998).

[22] W. Hillebrandt, R. G. Wolff, and K. Nomoto, Astron. \& Astrophys. 133, 175 (1984).

[23] G. 't Hooft, Phys. Lett. B 37, 195 (1971).

[24] S. Nakamura, T. Sato, S. Ando, T.-S. Park, F. Myhrer, V. Gudkov, and K. Ku- 
bodera, Nucl. Phys. A 707, 561 (2002), URL http://www.physics.sc.edu/ gudkov/NU-D-NSGK/

[25] E. Kolbe, K. Langanke, and P. Vogel, Nucl. Phys. A 652, 91 (1999).

[26] E. Kolbe, K. Langanke, and P. Vogel, Phys. Rev. D 66, 013007 (2002).

[27] E. Kolbe, K. Langanke, G. Martínez-Pinedo, and P. Vo- gel, J. Phys. G: Nucl. Part. Phys. 29, 2569 (2003).

[28] E. Kolbe and K. Langanke, Phys. Rev. C 63, 025802 (2001).

[29] K. Scholberg, in Proceedings of Neutrino 2006 (2007), arXiv:astro-ph/0701081. 